\title{
Contemporary challenges of warty carcinoma of cervix - Our experience and
}

\section{review of literature}

\section{Angel Yordanov ${ }^{1}$, Strahil Strashilov², Milena Karcheva ${ }^{3}$, Martin Karamanliev $^{4}$, Stanislav Slavchev ${ }^{5}$,} and Polina Vasileva ${ }^{6}$

1. Clinic of Gynecologic Oncology, Medical University Pleven, Bulgaria

2. Department of Plastic Restorative, Reconstructive and Aesthetic Surgery, Medical University Pleven,

Bulgaria

3. Department of Epidemiology, Medical University Pleven, Bulgaria

4. Department of Surgical Oncology, Medical University Pleven, Bulgaria

5. Clinic of Gynaecology, University Hospital "St. Anna"-Varna, Bulgaria

6. Department of Obstetrics and Gynecology, Medical University Pleven, Bulgaria

\section{RESEARCH}

Please cite this paper as: Yordanov A, Strashilov S, Karcheva M, Karamanliev M, Slavchev S, Vasileva P. Contemporary challenges of warty carcinoma of cervix - Our experience and review of literature. AMJ 2018;11(9):474-477.

https://doi.org/10.21767/AMJ.2018.3503

\section{Corresponding Author:}

Angel Danchev Yordanov

Clinic of Gynecologic Oncology, University Hospital "Dr. Georgi Stranski"-Pleven, Georgi Kochev 8A, Bulgaria Email: angel.jordanov@gmail.com

\section{ABSTRACT}

\section{Background}

Warty carcinoma of the cervix is a rare subtype of squamous cell carcinoma. In general, it is not as aggressive as the other subtypes, and has a better prognosis.

\section{Aims}

The aim of this study was to investigate overall and recurrence-free survival rate in patients with Warty carcinoma of the cervix.

\section{Methods}

During the ten year period (2008-2017) in the Clinic of Gynaecologic oncology at the UMHAT - Pleven, Bulgaria were operated 714 cases with cervical cancer, 14 of which were histologically confirmed as a Warty carcinoma. Patients were investigated by retro- and prospective analysis for overall and recurrence-free survival rate.

\section{Results}

Warty carcinoma accounts for 1.94 per cent of all cervical carcinomas, operated in the clinic. The mean age of the patients was 48 years, ranging from 29-72 years. According to the FIGO staging systems for cervix, patients were staged as follows: in stage IB1 - 43 per cent and in stage IB2 - 57 per cent. Despite the high percentage of locally advanced process, only in one case out of all the patients there was local spreading of the lesion towards the uterine cavity and in one case there were metastases in the pelvic lymph nodes. Lymphovascular space invasion was not seen in any patient, and neither were perineural or perivascular invasion.

\section{Conclusion}

This report highlights a favourable course and good prognosis of Warty carcinoma of the cervix. It is necessary to reconsider the aggressive surgical treatment and subsequent chemotherapy in women with Warty carcinoma of the cervix.

\section{Key Words}

Warty carcinoma of the cervix, survival rate, treatment

\section{Implications for Practice:}

\section{What is known about this subject?}

Warty carcinoma is one of the rare forms of squamous cell carcinoma of the uterine cervix and has better prognosis than usual SCC.

\section{What new information is offered in this case study?} Despite the high percentage of locally advanced process 
(patients with tumour above $4 \mathrm{~cm}$ ), there was no lymphovascular space invasion seen in any patient.

3. What are the implications for research, policy, or practice?

There is no need of aggressive surgical treatment in patients with warty cervical cancer, especially when tumour size is between 2 and $4 \mathrm{~cm}$.

\section{Background}

Warty carcinoma is one of the rare forms of squamous cell carcinoma (SCC). ${ }^{1,2}$ Although the SCC has many different types with different tumour biology, according to the world standards all the types of SCC are treated the same way. This histological type of carcinoma develops in principle from the stratified keratinized epithelium of the skin, the stratified squamous epithelium of the oral cavity, the oesophagus, the vagina. Carcinoma can also occur in places where there is no squamous epithelium - the lining of the bronchi, biliary tract, digestive tract, uterine body and others on the basis of pre occurring squamous metaplasia of the epithelium of these mucous membranes. The most common location of this tumour is in the anal and genital area - the vulva, vagina and uterine cervix, anus and penis. ${ }^{3}$ Certain risk factors for the development of cervical cancer are known - infection with human papilloma virus (HPV) types, smoking, and use of oral contraceptives for a long time, poverty and poor socio-economic status. ${ }^{4-11}$ The role of etiological factor in the development of warty carcinomas is proven for sure only in vulvar and penis localisation. ${ }^{2,12-18}$

\section{Method}

A combined retrospective and prospective cohort study of all cases of warty carcinoma of the cervix at Department of Gynaecologic Oncology, University Hospital - Pleven, Bulgaria between January 2008 and June 2018 was performed. Retrospective period was from January 2008 to December 2015 and prospective - from January 2016 to June 2018. We studied the relation of the occurrence of carcinoma to the menopause, the size of the tumour, the presence of local and distant metastases. The follow-up was done on $3,6,9,12,15,18,21,24$ months and then annually including clinical examination, blood tests and chest X-ray. Annually a whole-body contrast-enhanced CT was done. We looked at the time to tumour recurrence or occurrence of symptoms as well.

\section{Results}

During the study period 14 patients of 714 were recorded, which represent 1.94 per cent of all cervical carcinomas, operated in the clinic during the same period. In all patients a radical hysterectomy with total pelvic lymph node dissection with postoperative Tele-gama therapy (TGT) was performed, as this is the standard treatment for this disease in Bulgaria. The mean age of the patients was 48 years, ranging from $29-72$ years. Out of those, 57 per cent $(n=8)$ were peri- and postmenopausal women and 43 per cent $(n=6)$ were in women under the age of 45 . According to the FIGO staging systems for cervix, patients were staged as follows: in stage IB1 - 43 per cent $(n=6)$ and in stage IB2 57 per cent $(n=8)$. In 29 per cent $(n=4)$ the tumour size was under $2 \mathrm{~cm}$, in 14 per cent $(n=2)$ the size was $2-4 \mathrm{~cm}$ and in 57 per cent $(n=8)$ the size of the local tumour is above $4 \mathrm{~cm}$ (Table 1).

\section{Table 1: Clinical date of patients with warty carcinoma}

\begin{tabular}{|l|l|}
\hline Patients' clinical date & Number of patients \\
\hline Age & 14 \\
$<45$ years years & 6 \\
& 8 \\
\hline FIGO stage & 14 \\
IB1 & 6 \\
IB2 & 8 \\
\hline The size of the tumor & 16 \\
$<2 \mathrm{~cm}$ & 4 \\
$2-4 \mathrm{~cm}$ & 2 \\
$>4 \mathrm{~cm}$ & 8 \\
\hline
\end{tabular}

Despite the high percentage of locally advanced process (patients with tumour above $4 \mathrm{~cm}$ ), only in one case out of all the patients there was local spreading of the lesion towards the uterine cavity and in one case there were metastases in the pelvic lymph nodes. Lymphovascular space invasion was not seen in any patient, and neither were perineural or perivascular invasion. None of the patients has recurrence of the tumour or reoccurrence of symptoms. The maximum follow-up after surgery was 115 months and the minimum - 4 months. Incidence rate was 0 cases per 87.5 person years.

\section{Discussion}

Warty carcinoma of uterine cervix is a rare histologic type and it is poorly documented in the literature. It has two components -condyloma and invasive squamous cell tumour. The name "warty" derives from the appearance of the tumour - condylomatous. The diagnosis of "warty carcinoma" is histological and is based on a characteristic microscopic picture according to World Health Organization (WHO) - a typical feature of warty carcinoma is the 
presence of large numbers of atypical koilocytes, in the superficial and intermedia area of the epithelium and the microscopic examination reveals a biphasic pattern composed of a condylomatous and papillary configuration at the surface and SCC at the bottom. It seems that the malignant foci are directly transformed from condyloma. ${ }^{4}$ Warty carcinoma has to be differentiated from condylomata acuminate and verrucous carcinoma by its feathery surfacestout and smooth keratotic surface. Verrucous carcinoma has characteristic bulbous rete pegs deep in the stroma and a complete absence of koilocytes. ${ }^{4}$

The incidence of warty carcinoma in uterine cervix and other localizations is generally higher in peri- and postmenopausal women, but vulvar localization is more often in younger women. ${ }^{3}$ However, 45 per cent of our patients with warty carcinoma of the cervix are premenopausal. No specific preference for any race or ethnic group is noted. Usually, there are no symptoms, during the pre-cancer and early cancer stages. As the disease progresses the patients complain of abnormal vaginal bleeding, postcoital vaginal leading, menstrual cycle disturbances, loss of weight, loss of appetite, frequent urination, lower back pain, swollen lymph nodes in the groin. As a clinical behaviour, it stands between the verrucose and the low grade squamous cell carcinoma. It is usually found in postmenopausal women and have a better prognosis than the common cervical cancer. However this histologic subtype has metastatic potential when it affects other organs - for example the penile warty give metastasis in 17-18 per cent and there are metastasis in the vulvar area too. ${ }^{19}$ Cervical warty lesions are usually found only in loco and have no expansion toward other anatomical structures. Guidelines of ESMO и NCCN for treatment of cervical warty carcinoma recommend - radiotherapy (RT) and surgery are equally effective in early stages, so surgery should only be considered in patients with earlier stages (up to FIGO IIA) without risk factors necessitating adjuvant therapy, which results in a multimodal therapy without improvement of survival but increased toxicity. ${ }^{20,21}$ If the patient's fertility is to be preserved, an organ-saving operation may also be performed - radical trachelectomy with pelvic lymph dissection with or without paraaortic lymph node assessment. However, only female staged IB1 with a tumour size of up to $2 \mathrm{~cm}$ is suitable for this procedure.

Regardless of the size of the tumour, there is no case of warty carcinoma of the cervix with involvement of the parametric ligaments. Despite the potential for local and lymphovascular metastasis, single such cases have been described with a good prognosis for the patient. As far as we know, there are only two reported cases of warty carcinoma of uterine cervix spreading to the uterine body, ${ }^{22}$ one of which is described by our team and the another case is with left paraovarian metastasis, a pouch of Douglas metastasis and right paratubal metastasis. ${ }^{3}$ This would allow the widening of indications for organ-saving surgical procedures and surgical treatment towards total laparoscopic hysterectomy or modified radical hysterectomy with sentinel lymph node biopsy.

In the literature there is only one case with metastatic lymph nodes: two micrometastases of pelvic lymph nodes when total pelvic lymph dissection was performed (41 lymph nodes removed) described by our team. The tumour was over $4 \mathrm{~cm}$. Up to this date (65 months), the patient is alive and well, and has no recurrence. According to this finding, the lymph node metastasis may not affect the survival rate. To the best of our knowledge, this is the first study of patients with cervical localization of warty carcinoma for such period and providing such long followup.

\section{Conclusion}

In conclusion, warty carcinoma has a favourable progress and a good prognosis. Despite the theoretical possibility to metastasize, single such articles are reported in which, however, there are no change in the overall survival and free of recurrence survival. The data available to date may suggest rethinking of aggressive surgical behaviour in patients with this histological type of cervical cancer, especially when tumour size is between 2 and $4 \mathrm{~cm}$. To prove that larger randomized clinical trials are needed.

\section{References}

1. Wright TC, Ferenezy A, Kurman RJ. Carcinoma and other tumours of the vulva. (4th Edition) Springer-Verlag, New York, USA: 1994.

2. Kurman RJ, Norris HJ, Wilkinson EJ. Atlas of Tumour Pathologyy. Armed Forces Institute of pathology. Washington DC, USA: 1992.

3. Padberg BC, Bode B, Zimmermann D. Metastatic Warty (Condylomatous) Carcinoma of the Uterine Cervix Associated with Low-Risk HPV Type 6. The journal of clinical cytology and cytopathology Acta Cytol. 2006;50(2):235-8.

4. Cho $\mathrm{NH}$, Joo HJ, Ahn $\mathrm{HJ}$, et al. Detection of human papillomavirus in warty carcinoma of the uterine cervix: comparison of immunohistochemistry, in situ hybridization and in situ polymerase chain reaction methods. Pathol Res Pract. 1998;194:713-720.

5. Vilmer C, Cavelier-Balloy B, Nogues C, et al. Analysis of 
alterations adjacent to invasive vulvar carcinoma and their relationship with the associated carcinoma: a study of 67 cases. Eur J Gynaecol Oncol. 1998;19:25-31.

6. Petry KU, Kochel $\mathrm{H}$, Bode $\mathrm{U}$, et al. Human papillomavirus is associated with the frequent detection of warty and basaloid high-grade neoplasia of the vulva and cervical neoplasma among immunocompromised women. Gynecol Oncol. 1996;60:30-34.

7. Trimble $\mathrm{CL}$, Hildesheim $\mathrm{A}$, Brinton $\mathrm{LA}$, et al. Heterogeneous etiology of squamous carcinoma of the vulva. Obstet Gynecol. 1996;87:59-64.

8. Monk BJ, Burger RA, Lin F, et al. Prognostic significance of human papillomavirus DNA in vulvar carcinoma. Obstet Gynecol 1995;85:709-715.

9. Williams GR, Lu QL, Love SB, et al. Properties of HPV positive and HPV-negative anal carcinomas. J Pathol. 1996;180:378-382.

10. Ng WK, Cheung LK, Li AS. Warty (condylomatous) carcinoma of the cervix. A review of 3 cases with emphasis on thin-layer cytology and molecular analysis for HPV. Acta Cytol. 2003;47(2):159-66.

11. Rastkar G, Okagaki T, Twiggs LB, et al. Early invasive and in situ warty carcinoma of the vulva: clinical, histologic, and electron microscopic study with particular reference toviral association. Am J Obstet Gynecol. 1982;143:814820.

12. Jang YH, Kim YC, Lee ES. Warty squamous cell carcinoma of the culva in older women: Association with human papillomavirus. Yonsei Medical Journal. 2005;46:155158.

13. Al-Ghamdi A, Freedman D, Miller D, et al. Vulvar squamous cell carcinoma in young women: a clinicopathologic study of 21 cases. Gynecol Oncol. 2002;84:94-101.

14. Tarbunou Y, Davis C, Costa J, et al. Warty condylomatous squamous cell carcinoma of the penis in a 19-year-old. Urol Case Rep. 2014;2(3)79-81.

15. Chaux A, Lezcano $C$, Cubilla $A L$, et al. Comparison of subtypes of penile squamous cell carcinoma from high and low incidence geographical regions. Int J Surg Pathol. 2010;18(4):268-77.

16. Cubilla AL, Velazques EF, Reuter VE, et al. Warty (condylomatous) squamous cell carcinoma of the penis: a report of 11 cases and proposed classification of 'verruciform' penile tumors. Am J Surg Pathol. 2000;24(4):505-12.

17. Cabrero I, Sanchez D, Piedras D, et al. The variable morphological spectrum of penile basaloid carcinomas: differential diagnosis, prognostic factors and outcome report in 27 cases classified as classic and mixed variants. Applied Cancer Research. 2017;37:3.
18. Bezerra AL, Lopes A, Landman G, et al. Clinicopathologic features and human papillomavirus DNA prevalence of warty and squamous cell carcinoma of the penis. Am J Surg Pathol. 2001;25:673-8.

19. de Silva MVC, Fernando MS, Constantinem R, et al. Warty (condylomatous) carcinoma of the cervix, a variant of invasive squamous cell carcinoma with less aggressive behavior. J Obstet Gynyecol. 2001;22:31-32.

20. https://www.esmo.org/Guidelines

21. https://www.nccn.org/professionals/physician_gls/defa ult.aspx

22. Landeyro J, Gutierrez-Fornes C, Sirvent JJ. Warty (Condylotous) Carcinoma of the Cervix with extension to the endometrial cavity. Int J Gynaecol Obstet. 2011;112(1):66-7.

\section{PEER REVIEW}

Not commissioned. Externally peer reviewed.

\section{CONFLICTS OF INTEREST}

The authors declare that they have no competing interests.

\section{FUNDING}

This publication is supported by Project N BG05M2OP0012.009-0031-C01

\section{PATIENT CONSENT}

The authors, Yordanov A, Strashilov S, Karcheva $M$, Karamanliev $M$, Slavchev $S$, Vasileva $P$, declare that:

1. They have obtained written, informed consent for the publication of the details relating to the patient(s) in this report.

2. All possible steps have been taken to safeguard the identity of the patient(s).

3. This submission is compliant with the requirements of local research ethics committees. 